\title{
Coxibs were not cost effective for arthritis pain in patients with average risk of ulcer complications
}

\section{Spiegel BM, Targownik L, Dulai GS, et al. The cost-effectiveness of cyclooxygenase-2 selective inhibitors in the management of chronic arthritis. Ann Intern Med 2003;138:795-806. \\ QUESTION: In patients with osteoarthritis or rheumatoid arthritis, does the reduction in gastrointestinal (Gl) complications seen with coxibs offset their increased cost compared with non-steroidal anti-inflammatory drugs (NSAIDs)?}

\section{Design}

Cost utility analysis using a decision analytic model from the perspective of a third party payer considering direct medical costs.

\section{Setting}

USA.

\section{Patients}

A hypothetical cohort of patients 60 years of age with osteoarthritis or rheumatoid arthritis who were not taking aspirin and required NSAID therapy for moderate to severe arthritis pain.

\section{Intervention}

Patients who entered the model were treated with a coxib (celecoxib, $200 \mathrm{mg}$ once daily, or rofecoxib, $25 \mathrm{mg}$ once daily) or a non-selective NSAID at the maximum dose approved by the US Food and Drug Administration (naproxen, $500 \mathrm{mg}$ twice daily). The model was designed to test the hypothesis that coxibs are cost effective alternatives to NSAIDs.

\section{Main cost and outcome measures}

A decision tree was constructed to represent the coxib and naproxen strategies. Clinical probability estimates were derived from a systematic review of the literature using Medline and hand searching 2 subspecialty journals (January 1985 to December 2002). Validated utilities were assigned for GI outcomes: 0.87 for severe dyspepsia, 0.91 for moderate dyspepsia, 0.49 for ulcer haemorrhage, and 0.46 for complicated ulcer requiring surgery. The main outcome was the incremental cost per quality adjusted life year (QALY) gained, expressed in 2002 US dollars. All costs and outcomes were discounted at an annual rate of 3\%. Explicit assumptions were made to bias the analysis in favour of the coxib strategy. Both deterministic and probabilistic sensitivity analyses were done.

\section{Main results}

In the base case analysis, the coxib strategy had an incremental cost of \$275809 per additional QALY compared with the NSAID strategy. When a cohort of high risk patients (previous ulcer haemorrhage) was evaluated, the incremental cost effectiveness ratio decreased to $\$ 55803$ per QALY. Sensitivity analysis showed the model was sensitive to the cost per coxib pill, number of coxib pills consumed daily, cost per naproxen pill, and the probability of upper GI complications with naproxen.

\section{Conclusions}

In average risk patients with osteoarthritis or rheumatoid arthritis, the reduction in gastrointestinal complications seen with coxibs did not offset their increased costs compared with non-steroidal anti-inflammatory drugs. Coxibs were more cost effective in patients at increased risk of gastrointestinal complications.

\section{COMMENTARY} resources mandate consideration of cost in management decisions. is considered too expensive. NSAID use is lower than many would accept. increase in risk of ulcer complications is the threshold for cost savings. Rheumatol Suppl 1998:51:8-16. nonselective NSAID or coxib use. Gastroenterology 2003;124:28-92. rheumatoid arthritis. Gastroenterology 2002;1 23:1006-12.
Sources of funding: National Institutes of Health and Veterans Administration

For correspondence: Dr I M Gralnek,

Veterans

Administration Greate Los Angeles Healthcar System, Los Angeles, CA,USA igralnek@ mednet.ucla.edu

Abstract and commentary also appear in $A C P$ Journal Club

Cyclo-oxygenase 2 specific inhibitors (coxibs) cause fewer symptomatic ulcers, GI complications, and upper GI symptoms than non-selective NSAIDs. Although clinical and quality of life (QOL) outcomes are the primary focus of physicians and patients, limited

Cost effectiveness analyses assess expected costs per clinical outcome (or QOL adjusted outcome). When a new drug is safer or more effective but more expensive than standard therapy, 3 possible situations may occur: (1) the drug is cost saving because higher drug costs are outweighed by decreased medical costs because of better outcomes (eg, fewer hospital admissions); (2) overall costs are increased but the extra cost is considered worth the benefit; or (3) the additional cost for an improved outcome

The meticulous analysis by Spiegel et al indicates that the cost per QALY gained with coxibs is unacceptably high in average risk patients. Use of QALYs is recommended in cost effectiveness studies to allow comparisons across disparate interventions (eg, is it better to invest in childhood vaccination or liver transplantation?). However, since QALYs relate to duration and decrement in QOL, they may undervalue serious nonfatal acute illnesses (eg, hospital admission for ulcer bleeding was assigned only a 7 day QOL decrement in this analysis). The analysis also did not consider lower GI events, which may account for about $15-40 \%$ of all GI complications and seem to be decreased with coxibs. ${ }^{1-2}$ Furthermore, the estimated $10.9 \%$ lifetime probability of dyspepsia with

Nevertheless, the conclusions of Spiegel $e t$ al are certainly qualitatively correct. Use of coxibs in average risk patients is unlikely to achieve traditional levels of "cost effectiveness" at current US prices. As the risk of GI events increases, the number of patients needed to treat (NNT) to avert an additional upper GI event decreases (eg, age $<65 \mathrm{y}$, $\mathrm{NNT}=66$; age $>75$, NNT $=10),{ }^{3}$ and coxibs become more cost effective. In the highest risk patient, coxibs may actually be cost saving: Spiegel et al report that a 5.6 fold

Loren Laine, MD University of Southern California Los Angeles, California, USA

1 Singh G, Rosen Ramey D. NSAID induced gastrointestinal complications: the ARAMIS perspective-1997. Arthritis, Rheumatism, and Aging Medical Information System.

2 Laine L, Connors LG, Reicin A, et al. Serious lower gastrointestinal clinical events with

Laine L, Bombardier C, Hawkey CJ, et al. Stratifying the risk of NSAID-related upper gastrointestinal clinical events: results of a double-blind outcomes study in patients with 